\title{
Measurement of the electron electric dipole moment using YbF molecules
}

\author{
J.J. Hudson, B.E. Sauer, M.R. Tarbutt, and E.A. Hinds \\ Sussex Centre for Optical and Atomic Physics, University of Sussex, Brighton BN1 9QH, UK
}

(Dated: October 22, 2018)

\begin{abstract}
The most sensitive measurements of the electron electric dipole moment $d_{e}$ have previously been made using heavy atoms. Heavy polar molecules offer a greater sensitivity to $d_{e}$ because the interaction energy to be measured is typically $10^{3}$ times larger than in a heavy atom. We report the first measurement of this kind, for which we have used the molecule YbF. Together, the large interaction energy and the strong tensor polarizability of the molecule make our experiment essentially free of the systematic errors that currently limit $d_{e}$ measurements in atoms. Our first result $d_{e}=(-0.2 \pm 3.2) \times 10^{-26} \mathrm{e} \mathrm{cm}$ is less sensitive than the best atom measurement, but is limited only by counting statistics and demonstrates the power of the method.

PACS numbers: 13.40.Fn, 11.30.Er, 14.60.Cd, 39.20.+q, 33.15.Kr
\end{abstract}

The permanent electric dipole moment (edm) of an elementary particle vanishes unless the discrete symmetries parity $(\mathrm{P})$ and time reversal $(\mathrm{T})$ are both violated [1]. This naturally makes the edm small in fundamental particles of ordinary matter. In the standard model of elementary particle physics, a cancellation of leading order Feynman diagrams further suppresses the expected value of the electron edm $d_{e}$ to less than $10^{-38} \mathrm{e} \mathrm{cm} \mathrm{[2,}$ but in theories going beyond the standard model, typical predictions are in the range $10^{-26}-10^{-28} \mathrm{ecm}[3$. The search for an electron edm is therefore a search for physics beyond the standard model, and particularly it is a search for non-standard $\mathrm{CP}$ violation. $\mathrm{CP}$ violation is intimately connected to T-violation through the CPT theorem 位. This is an important and active field at present [5] because the prospects for discovering new physics seem so good. Such research is complementary to experiments on $\mathrm{K}$ and $\mathrm{B}$ particles where the observed $\mathrm{CP}$ violation confirms the standard model CKM mechanism [6]. The most precise measurement of the electron edm is that of Regan and Commins [7], who have achieved the result $d_{e}=(7 \pm 8) \times 10^{-28}$ e cm by searching for a differential Stark shift between the two hyperfine sublevels $\left(F=1, m_{F}= \pm 1\right)$ in the ground state of atomic thallium. Their result imposes stringent constraints on possible theories of particle physics. Any improvement would make a very important contribution to the further search for new physics beyond the standard model, but their method seems to have reached its limit because of systematic errors associated with stray magnetic fields. Such errors should be greatly suppressed if $d_{e}$ could be measured using heavy polar molecules instead of atoms [8], but hitherto this has not been technically possible. Here we report on the first experiment to realize this idea, which demonstrates the feasibility of the method and, although not yet as accurate as the thallium experiment, nevertheless obtains the next most significant constraint [9] on $d_{e}$. We show that this new molecular method is potentially much more sensitive than the previous atomic experiments.
To leading order in $d_{e}$ the edm interaction of an electron in an atom or molecule can be described by an effective Hamiltonian $d_{e}\left(1-\gamma_{0}\right) \Sigma \cdot \mathbf{E}_{t o t}$, where $\gamma_{0}$ and $\Sigma$ are standard Dirac matrices and $\mathbf{E}_{t o t}$ is the total electric field at the position of the electron, including the field of the other electrons [10]. The explicit form of this interaction,

$$
V=\left\langle\psi_{0}\left|\begin{array}{cc}
0 & 0 \\
0 & 2 d_{e} \hat{\sigma} \cdot \mathbf{E}_{t o t}
\end{array}\right| \psi_{0}\right\rangle
$$

where $\hat{\sigma}$ is a unit vector along the electron spin, shows that the effect is relativistic: the operator connects only the small components of the Dirac wavefunction $\left|\psi_{0}\right\rangle$. The sensitivity to $d_{e}$ is therefore largest in heavy systems where the electrons are most relativistic. If there is no applied electric field, the small component of $\left|\psi_{0}\right\rangle$ has definite parity and therefore the expectation value of this odd-parity operator vanishes. However, an applied field $E \hat{\mathrm{z}}$ polarizes the wavefunction and yields an energy $V$ of the form $-d_{e} E_{e f f}\langle\hat{\sigma} \cdot \hat{\mathrm{z}}\rangle$. A strong laboratory field applied to an atom induces only a small mixing of atomic orbitals and therefore $E_{\text {eff }}$ is essentially proportional to $E$, with the ratio scaling roughly as $8 Z^{3} \alpha^{2}$ [11], $Z$ being the nuclear charge and $\alpha$ the fine structure constant. The cesium atom used by Hunter's group to measure $d_{e}$ 12 is a particularly favorable case with the applied field being effectively amplified by 119 [3]. Thallium has an even larger amplification of -585 , a key factor in the more sensitive result of ref. [7]. Diatomic polar molecules have a great advantage over atoms [8] because the atomic orbitals are naturally strongly polarized along the internuclear axis $\hat{\lambda}$. This gives rise to an internal effective electric field $E_{\text {int }} \hat{\lambda}$ that can be very large when one of the atoms is heavy and has strong s$\mathrm{p}$ hybridization of its orbitals. The molecular rotation averages this strong field to zero unless an external field $E \hat{\mathrm{z}}$ is applied. However, only a modest field is needed to polarize $\hat{\lambda}$ along $\hat{z}$ because this involves mixing rotational states, which are much closer together than the electronic states of an atom. The edm interaction energy $V$ once again takes the form $-d_{e} E_{e f f}\langle\hat{\sigma} \cdot \hat{z}\rangle$, but $E_{e f f}$ is 


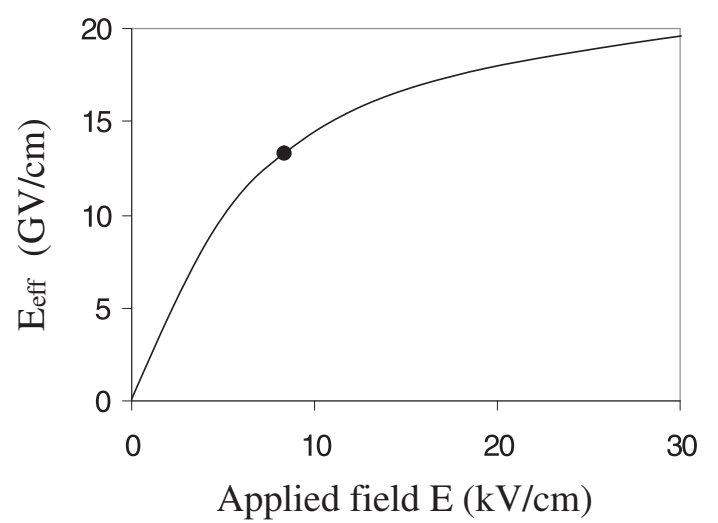

FIG. 1: Effective electric field interacting with the electron edm in YbF versus applied electric field. The dot shows the field at which we operate.

now given by $E_{\text {int }}\langle\hat{\lambda} \cdot \hat{z}\rangle$. Figure 1 shows $E_{\text {eff }}$ of the $\mathrm{YbF}$ molecule, rising towards its asymptotic value $E_{\text {int }}$ of $26 \mathrm{GV} / \mathrm{cm}$ [13] as the applied field is increased. In our experiment the applied field is $8.3 \mathrm{kV} / \mathrm{cm}$, for which $E_{\text {eff }}$ is $13 \mathrm{GV} / \mathrm{cm}$. Such a large effective field is not particular to $\mathrm{YbF}$ but can be found in a variety of other heavy polar diatomic molecules, some of which are listed in Table 1 . In short, the edm interaction in heavy polar molecules can be thousands of times larger than in heavy atoms.

Our experiment uses ${ }^{174} \mathrm{YbF}$ in the electronic, vibrational and rotational ground state $X^{2} \Sigma^{+}(v=0, N=0)$. The electron spin $(1 / 2)$ and the fluorine nuclear spin $(1 / 2)$ combine to produce a hyperfine singlet $F=0$ and triplet $F=1$, separated by $170 \mathrm{MHz}$ 17 as shown inset in fig. 2. The applied electric field lowers the energy of the $\left|F, m_{F}\right\rangle=|1,0\rangle$ state relative to $|1, \pm 1\rangle$ by an amount $\Delta(\Delta / h=6.7 \mathrm{MHz}$ for the $8.3 \mathrm{kV} / \mathrm{cm}$ used in our experiment) [18]. This strong tensor splitting reflects the non-spherical symmetry of the molecule's internal structure. The two states $|1,+1\rangle$ and $|1,-1\rangle$ remain degenerate as a consequence of time-reversal symmetry. Their degeneracy is lifted by the edm interaction, which causes a splitting of $2 d_{e} E_{e f f}$ that we seek to measure in the experiment. A magnetic field small

\begin{tabular}{cc}
\hline \hline Species: state & $E_{\text {eff }}(\mathrm{GV} / \mathrm{cm})$ \\
\hline $\mathrm{BaF}: \mathrm{X}^{2} \Sigma^{+}$ & $7.4^{(a)}$ \\
$\mathrm{YbF}: \mathrm{X}^{2} \Sigma^{+}$ & $26^{(b)}$ \\
$\mathrm{HgF}: \mathrm{X}^{2} \Sigma^{+}$ & $99^{(c)}$ \\
$\mathrm{PbF}: \mathrm{X}^{2} \Sigma^{+}$ & $-29^{(c)}$ \\
$\mathrm{PbO}: \mathrm{a}(1)^{3} \Sigma^{+}$ & $6^{(d)}$ \\
\hline \hline
\end{tabular}

TABLE I: Effective electric fields for some heavy polar molecules. ${ }^{a}$ ref. [14], ${ }^{b}$ ref. [13], ${ }^{c}$ ref. [15], ${ }^{d}$ ref. [16]

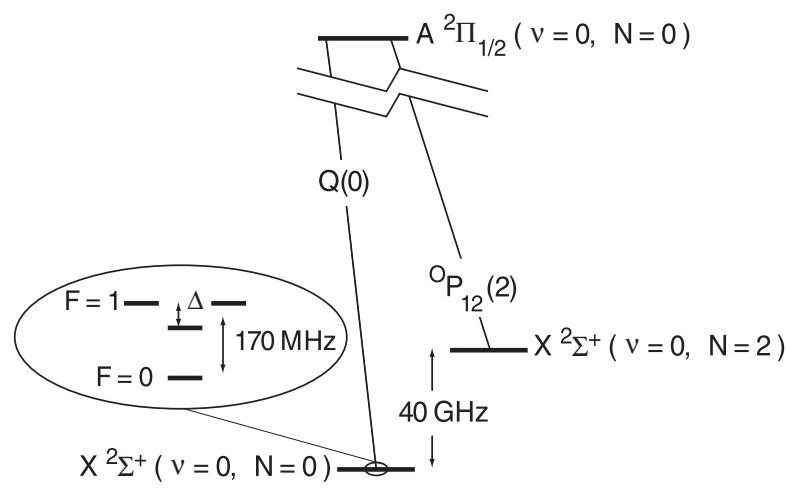

FIG. 2: Important optical transitions $\mathrm{Q}(0)$ and ${ }^{\mathrm{O}} \mathrm{P}_{12}(2)$ in ${ }^{174} \mathrm{YbF}$ at $553 \mathrm{~nm}$. They are $40 \mathrm{GHz}$ apart. Inset: ground state hyperfine levels $F=0, F=1,170 \mathrm{MHz}$ apart. In static electric field, the $m_{F}=0$ sublevel of $F=1$ is lower than the $m_{F}= \pm 1$ sublevels by an amount $\Delta$.

compared with $\Delta / \mu_{B}$ causes an additional splitting 19. of $2 \mu_{B} B_{z}\left(1-\frac{1}{2}\left(\mu_{B} B_{\perp} / \Delta\right)^{2}\right)$ plus higher order corrections (here the g-factor, both expected and measured, is $1)$. This formula shows that the field parallel to $\mathbf{E}$ induces a Zeeman splitting $2 \mu_{B} B_{z}$ between the $m_{F}= \pm 1$ sublevels, whereas the splitting due to the perpendicular field $B_{\perp}$ is suppressed relative to $\mu_{B} B_{\perp}$ by a factor $\mu_{B}^{2} B_{z} B_{\perp} / \Delta^{2}$, which is $3 \times 10^{-10}$ in our experiment $\left(B_{z} \approx 10 \mathrm{nT}, B_{\perp} \approx 6 \mathrm{nT}\right)$. We separate the splitting due to the edm interaction from that of the magnetic interaction by reversing the directions of the applied electric and magnetic fields, $\mathbf{E}$ and $\mathbf{B}$. The edm part of interest has the symmetry of $\mathbf{E} \cdot \mathbf{B}$, as one might expect for a P-odd, T-odd effect. The suppression of the splitting induced by $B_{\perp}$ is a critical aspect of the experiment because the motion of the molecules through the electric field generates a $6 \mathrm{nT}$ contribution to $B_{\perp}, B_{y}^{m o t}=E v / c^{2}$, which reverses with $\mathbf{E}$ and therefore has the potential to masquerade as an edm [20]. If $B_{\perp}$ is entirely motional it does not generate a false edm because the splitting depends on $B_{\perp}^{2}$, remaining unchanged when $B_{\perp}$ reverses. However, if there is also a small $y$-component $B_{y}$ of the applied magnetic field, the magnitude of $B_{\perp}$ will change when either $\mathbf{E}$ or $\mathbf{B}$ is reversed, leading to an apparent edm given by $\mu_{B}^{3} B_{z} B_{y}^{m o t} B_{y} / \Delta^{2} E_{e f f}$. In our experiment $B_{y}$ is less than $1 \mathrm{nT}$ and therefore this false $d_{e}$ is less than $10^{-33} \mathrm{e} \mathrm{cm}$. The advantage of a strong tensor polarizability for edm measurements was first demonstrated by Player and Sandars using the ${ }^{3} P_{2}$ metastable state of Xe [21. These two features of heavy polar molecules - large $E_{e f f}$ and strong tensor polarizability — give them such excellent suppression of all the known systematic errors that a major improvement in $d_{e}$ now seems accessible.

Our YbF molecular beam, illustrated in fig. 3, effuses out of a molybdenum oven containing a mixture of $\mathrm{Yb}$ metal and powdered $\mathrm{AlF}_{3}$ (mass ratio 4:1) heated to $\sim 1500 \mathrm{~K}$. The molecules are detected by dye-laser- 


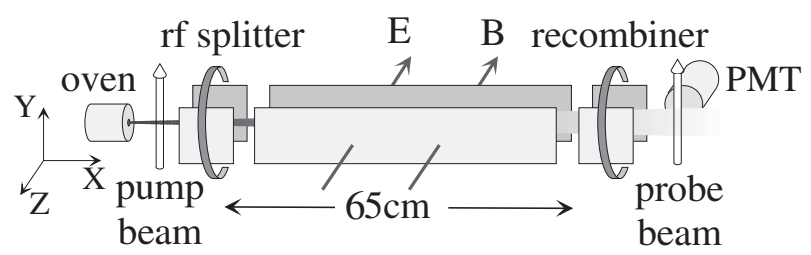

FIG. 3: Schematic of the YbF molecular beam apparatus.

induced fluorescence $1 \mathrm{~m}$ away from the source, using collection optics with $22 \%$ efficiency and a photomultiplier (PMT) of 10\% quantum efficiency. The detection laser is tuned to the $F=0$ component in the $\mathrm{Q}(0)$ line of the $\mathrm{A}^{2} \Pi_{1 / 2}-\mathrm{X}^{2} \Sigma^{+}$electronic transition (fig. 2) at $553 \mathrm{~nm}$. This induces a count rate of $2.5 \mathrm{kHz}$ due to the ${ }^{174} \mathrm{YbF}$ ground state $F=0$ molecules and $20 \mathrm{kHz}$ from nearby transitions involving higher rotational states and other isotopes of $\mathrm{Yb}$ [18. There are also backgrounds of $\sim 60 \mathrm{kHz}$ from oven light and $\sim 30 \mathrm{kHz}$ from scattered laser light. The pump beam of fig. 3 consists of two dye laser beams. One is a $170 \mathrm{MHz}$ red sideband of the probe laser. This removes molecules from all the sublevels of the $F=1$ ground state by exciting them to $\mathrm{A}^{2} \Pi_{1 / 2}$, which has negligible hyperfine structure 22. A second dye laser $40 \mathrm{GHz}$ to the red, tuned to the ${ }_{\mathrm{P}_{12}}(2)$ transition (fig. 2), excites molecules out of the highest hyperfine level of the $N=2$ rotational manifold. Together these two beams pump molecules into the $F=0$ ground state, increasing the probe signal to $8 \mathrm{kHz}$, while emptying out $F=1$. After pumping, the beam enters an electric field of $3.3 \mathrm{kV} / \mathrm{cm}$, where a $170 \mathrm{MHz}$ rf magnetic field along the $x$-direction excites the molecules to a coherent superposition $\frac{1}{\sqrt{2}}|1,+1\rangle+\frac{1}{\sqrt{2}}|1,-1\rangle$ of the $\left|F, m_{F}\right\rangle$ states. In essence this step is the beam splitter of a molecular interferometer. Next the molecules enter the center of the interferometer, where they evolve for a time $T(\sim 1 \mathrm{~ms})$ in combined electric and magnetic fields $( \pm E, \pm B) \hat{z}$, and the two parts of the wavefunction develop a relative phase shift of $2 \varphi=2\left( \pm d_{e} E_{\text {eff }} \mp \mu_{B} B\right) T / \hbar$. To measure this phase, a second rf loop drives a transition back to the $F=0$ state. This is the recombiner in fig. 3 . The state amplitude $\frac{1}{\sqrt{2}}\left(e^{i \varphi}+e^{-i \varphi}\right)$ gives a final $F=0$ population proportional to $\cos ^{2} \varphi$. Our detector, measuring fluorescence from molecules excited on the $\mathrm{Q}(0), F=0$ transition, probes this population. Fig. 4 shows the central interferometer fringe, measured by scanning the applied magnetic field. To obtain this fringe pattern it is necessary to suppress stray magnetic fields, which we do by means of two $1 \mathrm{~mm}$-thick layers of high-permeability (AdMU 80) magnetic shielding. We note that coherences at the $170 \mathrm{MHz}$ rf frequency play no role in this experiment; indeed the two rf loops are driven by 2 different oscillators to avoid any such Ramsey interferences.

The interferometer is most sensitive to small changes in phase when $\varphi= \pm \pi / 4$, i.e. when the magnetic field $\pm B_{0}$

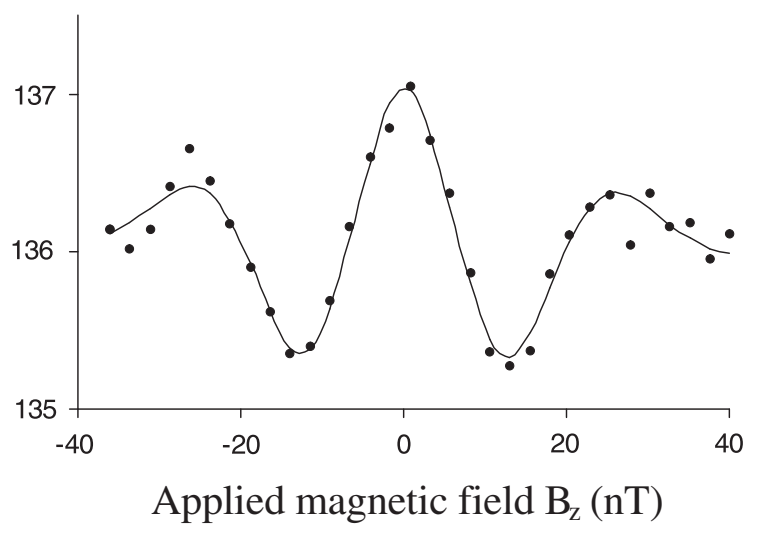

FIG. 4: Interference fringe in the $F=0$ state population versus magnetic field. Each point represents $12 \mathrm{~s}$ of integration time. The curve is a velocity-averaged calculation whose only free parameters are the normalization, a field offset, and a sloping background.

is set near the points of steepest slope at $\pm 6 \mathrm{nT}$ on either side of the central fringe. We record the fluorescence in the detector under the 8 conditions $\pm E, \pm\left(B_{0} \pm \delta B\right)$, where $\delta B$ is a carefully calibrated change of $1.6 \mathrm{nT}$ in the magnitude of the applied field. Over a period of 82 seconds these 8 measurements are repeated 128 times, ordered in such a way as to minimise drifts [23]. The duty factor is $60 \%$. This constitutes a "block" of data. In order to maintain operation near the most sensitive points (the residual field drifts by a few nT over a period of hours), we compare the PMT count rates for $+B_{0}$ and $-B_{0}$ (averaged over the other reversals) at the end of each block. Any difference indicates a nonzero residual field, which we null by adding a small proportional bias.

The dye in both lasers has a short lifetime ( $\sim 20$ hours $)$ as does the molecular beam source ( $\sim 10$ hours). Frequent maintenance and the small signal and large background in our detector make the experiment difficult to operate at present. Nevertheless, we have succeeded in taking 24 hours of data over a period of 4 months, a total of 1758 data blocks containing $1.1 \times 10^{10}$ counts. In our analysis of the data we extract the change in PMT count rate correlated with every combination of $E, B$ and $\delta B$ reversals within every block. Two of these combinations give the phase shift due to the edm interaction and the phase shift due to $\delta B$. Their ratio, $d_{e} E_{e f f} / \mu_{B} \delta B$, yields the edm result $d_{e}=(-0.2 \pm 3.2) \times 10^{-26} \mathrm{e} \mathrm{cm}$, where the uncertainty $(1 \sigma)$ is due entirely to random pulse counting statistics.

All other combinations of all the switched parameters are consistent with zero, suggesting that systematic errors are well under control. As a further check, we took the data in 4 sets with the connections to the electric plates and/or magnetic field wires reversed, and this too revealed no systematic errors. We monitored the leak- 
age current of the plates, which was always below $10 \mathrm{nA}$ on both plates during data taking. At worst, this current (implausibly looping around the edge of each plate) would cause a false edm of $4 \times 10^{-28} \mathrm{e} \mathrm{cm}$. This is small compared with the result of ref $[7]$ and is insignificant in the present experiment. With a view to future improvements in sensitivity, we checked the effect of a change in field magnitude when the electric field was reversed. This changed the resonance strength and therefore the slope of the fringe. When combined with an asymmetry of the magnetic field it led to a false edm, but one that we were fully able to understand and correct on the basis of the separate E-reversal and B-reversal signals. We also ran the experiment with $B_{\perp}$ intentionally amplified by a factor of 50 and saw that this did not induce any false edm at the level of $5 \times 10^{-26} \mathrm{ecm}$. All these effects were negligible in the present experiment and seem to be well under control for a future measurement below $1 \times 10^{-27} \mathrm{e} \mathrm{cm}$. Some details of the systematic checks are available in reference 19 .

This experiment has demonstrated that a molecular measurement of $d_{e}$ is less sensitive to stray magnetic fields than any previous experiment by several orders of magnitude. This is due to the high value of $E_{\text {eff }}$. The cylindrical symmetry of the electronic state also makes the molecule immune to the motional magnetic field effect. The present result could improve considerably with higher beam intensity. To this end, we have developed a new pulsed source in which $\mathrm{YbF}$ molecules are produced by laser ablation and cooled below $20 \mathrm{~K}$ by supersonic expansion in a jet of carrier gas. This produces 100 times more population in the $N=0$ rotational state. On the basis of its current performance, we expect that incorporating the supersonic source into our experiment will reduce the statistical uncertainty in $d_{e}$ to below $1 \times 10^{-27} \mathrm{ecm}$ in 24 hours. A competing experiment is in preparation at Yale University by the group of D. DeMille using a cell of $\mathrm{PbO}$ vapor excited to the paramagnetic metastable a(1) state 16]. Trapped cold atoms offer a possible alternative to using polar molecules for measuring $d_{e}$ [24]. They can have transverse spin relaxation times of seconds instead of milliseconds and the atoms move slowly making the $\mathbf{E} \times \mathbf{v}$ effect much less than in an atomic beam. Even so, the sensitivity to stray magnetic fields and to the trapping fields is a very severe problem for paramagnetic atoms and the experiment would have to use two trapped species, a heavy one for measuring $d_{e}$ and one for monitoring the magnetic field. Trapped cold molecules offer the best prospect of all, but at present the required techniques are only in a very early stage of development 25.

We are indebted to Alan Butler for his expert technical assistance. This work was supported by the EPSRC and
PPARC research councils of the UK.

[1] E.M.Purcell and N.F. Ramsey, Phys. Rev. 78, 807 (1950); L.D. Landau, Zh. Eksp. Theor. Fiz., 32, 405 (1957) [Sov. Phys. JETP 5, 405 (1957)].

[2] M. Pospelov and I.B. Khriplovich, Sov. J. Nucl. Phys. 53 638-640 (1991).

[3] A recent review of possible electron edm values can be found in E.D. Commins, Ad. At. Mol. Opt. Phys. 40, 1 (1999).

[4] See, for example, the discussion of the CPT theorem in Robert G. Sachs, The Physics of Time Reversal, (University of Chicago Press, 1987).

[5] Art and symmetry in experimental physics: Festschrift for Eugene D. Commins AIP conference proceedings 596, eds. D. Budker, P.H. Bucksbaum and S.J. Freedman (AIP New York 2001).

[6] L. Wolfenstein, Euro. Phys. J. 15, 115, (2000); Aubert et al., Phys. Rev. Lett. 87, 091801 (2001); Abe et al., Phys. Rev. Lett. 87, 091802 (2001).

[7] B.C. Regan, E.D. Commins, C.J. Schmidt and D. DeMille, Phys. Rev. Lett. 88, 071805 (2002).

[8] P.G.H. Sandars in Atomic Physics 4 edited by G. zu Putlitz, (Plenum, 1975) p.71.

[9] Unpublished constraints of similar precision can be derived from measurements on Cs and $\mathrm{Hg}$. L.R. Hunter and E.N. Fortson, private communication (2002).

[10] See $\S 4$ of [11] or $\S$ III of [3].

[11] E.A. Hinds, Physica Scripta T70, 34 (1997).

[12] S.A. Murthy et al., Phys. Rev. Lett. 63, 965 (1989).

[13] M.G. Kozlov, V.F. Ezhov, Phys. Rev. A49, 4502 (1994); M.G. Kozlov, J. Phys. B 30 L607 (1997); A. V. Titov, N. S. Mosyagin, V. F. Ezhov, Phys. Rev. Lett. 775346 (1996); H.M. Quiney, H. Skaane, I.P. Grant, J. Phys. B 31 L85 (1998) (after correcting for the trivial factor of 2 between $\mathbf{s}$ and $\sigma$ their result becomes $26 \mathrm{GV} / \mathrm{cm}$ ); F.A. Parpia, J. Phys. B 311409 (1998); N. Mosyagin, M. Kozlov, A. Titov, J. Phys. B 31 L763 (1998).

[14] M.G. Kozlov, A. V. Titov, N. S. Mosyagin, P. V. Souchko, Phys. Rev. A., 56, R3326 (1997).

[15] Y.Y. Dmitriev et al., Phys. Lett. A 167, 280 (1992).

[16] D. DeMille et al., Phys. Rev. A, 61, 052507 (2000). See also ref [5], p.72.

[17] B.E. Sauer, Jun Wang and E.A. Hinds, Phys. Rev. Lett. 74, 1554 (1995).

[18] B.E. Sauer, Jun Wang and E.A. Hinds, J. Chem. Phys. 105, 7412 (1996).

[19] J.J. Hudson, D.Phil. thesis, University of Sussex, Brighton, (2001).

[20] Sometimes known as the Aharonov Casher phase shift. See Karin Sangster et al., Phys. Rev. A 51, 1776 (1995).

[21] M.A. Player and P.G.H. Sandars, J. Phys B 3, 1620 (1970).

[22] B.E. Sauer et al., J. Chem. Phys. 110, 8424 (1999).

[23] G.E. Harrison, M.A. Player, and P.G.H. Sandars, J. Phys E 4, 750 (1971).

[24] C.Chin et al., Phys Rev. A, 63, 033401 (2001).

[25] B.G. Levi, Physics Today, (September 2000) p. 46. 